\title{
Exploring the Role of Creativity in Software Engineering
}

\author{
Wouter Groeneveld*, Laurens Luyten ${ }^{\dagger}$, Joost Vennekens ${ }^{\ddagger}$, and Kris Aerts* \\ ${ }^{*} K U$ Leuven, Diepenbeek Campus, Department of Computer Science, Belgium \\ ${ }^{\dagger}$ KU Leuven, Sint-Lucas Brussels and Ghent Campus, Department of Architecture, Belgium \\ ${ }_{\ddagger}^{\ddagger}$ KU Leuven, De Nayer (Sint-Katelijne-Waver) Campus, Department of Computer Science, Belgium \\ \{firstname.lastname\}@kuleuven.be
}

\begin{abstract}
In order to solve today's complex problems in the world of software development, technical knowledge is no longer enough. Previous studies investigating and identifying nontechnical skills of software engineers show that creative skills also play an important role in tackling difficult problems. However, creativity is typically a very vague concept to which everyone gives their own interpretation. Also, there is little research that focuses specifically on creativity in the field of software engineering. To better understand the role of creativity in this field, we conducted four focus groups, inviting 33 experts from four nationally and internationally renowned companies in total. This resulted in 399 minutes of transcripts, further coded into 39 sub-themes grouped into seven categories: technical knowledge, communication, constraints, critical thinking, curiosity, creative state of mind, and creative techniques. This study identifies the added value of creativity, which creative techniques are used, how creativity can be recognized, the reasons for being creative, and what environment is needed to facilitate creative work. Our ultimate goal is to use these findings to instill and further encourage the creative urge among undergraduate students in higher education.
\end{abstract}

Index Terms - creativity, professional skills, industry requirements, software engineering education

\section{INTRODUCTION}

As enterprise software development gets more and more complex, overcoming big hurdles takes more than just technical knowledge. In a recent Delphi study, important skills of exceptional software engineers were identified [1]. Industry experts agreed that being creative (e.g., by approaching a problem from different angles) is an essential problem solving skill, vital to succeed as a software developer. However, creativity is a multidimensional concept that is not as easy to define as one might think.

Software engineering (SE) is said to be an outcome of human knowledge and creativity [2]. Therefore, we wonder:

- Q1: What exactly is the role of creativity in the world of SE?

- 22: What makes one software developer very creative and the other less so?

Even though cognitive creativity and creative behavior are well-researched in the field of psychology [3], [4], it is still difficult to answer these questions specifically for software development. As Amin et al. concluded in their systematic literature review: "The research work on creativity in $S E$ is scattered and scarce" [5]. To shed more light on this subject, we explore the role of creativity using focus groups as a way to gather qualitative data from experts in the industry. It is important to note that there are dozens of definitions of 'creativity' in literature. Rather than using an existing definition as a guideline, our intention is to explore what is understood by the term creativity, according to software engineers.

Our goal for this research is to contribute to narrowing the gap between higher education and the requirements of industry, as creativity is required of engineers to solve complex problems and research has shown that creativity is currently underrepresented in higher education computing curricula [6].

The remainder of this paper is divided into the following sections. Section 2 describes related work, while section III clarifies the utilized methodology. Next, in section 4, we present and discuss the results of the focus groups, followed by limitations in section 5. Section 6 concludes this work.

\section{RELATED WORK}

John Gero describes creative design by comparing it to innovative and routine design [7]. He defines routine design in computational terms as an activity which occurs when all necessary knowledge is a priori available: a pre-established procedure can be followed to come to a design solution. In contrast, he defines non-routine design by two subgroups: innovative and creative design. In innovative design the value of the variables directing the procedure to establish an outcome, are placed outside the intended range. This leads to design outcomes that are new but still belong to the same class as their routine progenitors. In creative design one or more new variables are introduced in the process leading to an all together new class of design outcomes.

In the field of cognitive psychology, countless creative models have been proposed in literature, of which Amabile's [8] (Expertise, Creative Thinking Skills, Motivation) and Mooney's 4P [9] (Process, Product, People, Place) certainly are the most popular. These models have been well-researched in context of varying fields, including SE [5]. One of the disadvantages of starting out with such a model is perhaps the biased view of creativity, focusing on only one of the four P's instead of exploring all possibilities, as our intention is. 
Requirements engineering is one of the more popular software-related research areas where creativity has been studied. In an effort to better understand problem solving in requirements engineering, Cybulski et al. discussed creativity with practitioners using focus groups [10]. According to the authors, 'requirements engineering is well-recognized as a creative problem solving activity by the systems development community'. The framework utilized to guide the focus groups is reminiscent of Csikszentmihalyi's three-dimensional systems view of creativity [11]: context (individual and social dimensions), outcome (the development of creative information systems), and process (supporting the creative characteristics of problem solving) form the main elements of creative problem solving. However, requirements engineering is not software engineering, and therefore, the findings grouped in these three dimensions might not be readily applicable in context of enterprise software development.

'What makes a great software engineer?' is a question that Paul Luo Li answered in his 2016 dissertation [12]. Good software engineers are essential to the creation of good software. Instead of asking what makes a software developer creative and the other less so, Luo Li asked what makes a software developer great and the other less so. The answer is, among others, the ability to be creative: applying novel and innovative solutions based on understanding the context and limitations of existing solutions. In interviews with industry experts, 'getting a little creative' is an often-returning expression. Sadly, the exact role of creativity itself is never further explored. We can conclude that creativity indeed plays an important role in solving programming problems, but we still do not know how creativity is manifested.

In 2019, Anna E. Bobkowska explored creativity techniques in SE using a specific training-application-feedback cycle [13]. Since creativity research has produced more than a hundred different techniques, the question becomes whether or not any of these techniques are applicable to the field of SE. Seven techniques were explored: naive questions (1; discover hidden assumptions and implicit knowledge), reverse brainstorming (2; first express criticism, then motivate to improve), Lunette (3; look at the problem at different levels of abstraction), Chinese dictionary (4; a technique to create atypical classifications), What if... (5; search for hidden sequences of consequences), I could be more creative if... (6; understand personal obstacles), and Let's invite him/her (7; use creativity patterns of experts in creativity). Participants left the experiment with an increased appreciation for creativity techniques, claiming that a mix of these techniques is likely to be useful in practice. However, instead of starting from certain techniques and matching them to software development, our aim is to approach creativity with an open mindset and let the answers come from the participants instead of the literature.

There seem to be few studies that explore creativity in context of the practice of SE. Instead, we mostly come across theoretical considerations. Amin et al. are right: the research work on creativity, specifically geared towards SE, is indeed scattered and scarce. In order to approach the research questions with an open mind, we invited 33 experts from the industry to discuss creativity, as explained in the following section.

\section{METHODOLOGY}

We conducted four focus groups, collecting information from developers from different agile software development companies. A focus group is a small group of experts in a specific field, who brainstorm about a specific subject in guided open discussions, to gain a better understanding on the subject at hand [14]. In this case, experts are experienced developers and the subject is creativity.

The focus group method was adapted for SE based on the work of Kontio et al. [15], as they state it "is best suited to obtain feedback on new concepts, [...] generating ideas". To simplify the organization and to increase the likely-hood of participating, each session was held at a separate company. Instead of using purposive sampling techniques to compose the focus groups, they were formed by using preexisting groups, namely colleagues. According to Fern, this is not bias but rather an advantage [16], since it has the added advantage that participants feel comfortable as they know each other. Due to the COVID-19 pandemic, the last two sessions were held online.

\section{A. The Focus Group Process}

Expert selection was done by limiting participation to people with a technical role that come into daily contact with source code, such as developers, programmers, software architects, ... To further increase relevance of answers, experts were required to have at least six years of experience in the field, and should be intrinsically motivated to partake in the discussion. Participation was entirely voluntary. Note that, although the job title of many participating experts is developer, this does not mean that their job is limited to merely implementing pre-set requirements. The four agile software development companies involved define the role developer as someone who is involved in both analysis, design, implementation, support, and maintenance phases of a software project.

The conversations were facilitated by the first author, since he has more than 11 years of experience in the industry. Even if some researchers claim that familiarity with the topic will introduce bias [16], Kontio et al. state that "In the field of $S E$, it is very important for the interviewer to have extensive knowledge of the theme of the interview" [15]. To reduce bias as much as possible, each session was audio-recorded, transcribed, and cross-validated by the second author, whose field of expertise is architecture rather than SE.

During two hours, open questions are used to elicit opinions, perceptions, and ideas, which further help us as researchers to determine the role of creativity in software development. In order not to influence the results, we chose to refrain from providing a clear definition of creativity. By not defining the concept ourselves, we try to find out what the industry might mean when they seek "creative" software engineers. The following questions were addressed: 
1. What is the most creative thing you ever did in your job?

2. How can you see when a colleague is being creative, or not?

3. What is the most creative project you ever worked on?

4. How could you measure how creative someone is?

5. Which creative techniques did you recently employ?

6. What is the most important reason to be creative?

7. Which environment do you need to be creative?

As an ice-breaker, participants first had to complete the sentences "as a developer, I am creative when I ..." (1) and "as a developer, I am not creative when I ..." (2). After this individual warm-up exercise, the facilitator gave a brief overview of Amabile's Componential Model [8] (Expertise, Creative Thinking Skills, Motivation) and Mooney's 4P Model [9] (Process, Product, People, Place) to increase the awareness of the multi-dimensional aspects of creativity in general. Next, the above questions were asked in random order, where participants were grouped in pairs to brainstorm for five minutes, followed by ten minutes of discussion in group.

\section{B. Processing Focus Group Data}

Data was identified by concepts that 'bubble up' and present themselves after multiple data gathering and reduction steps, as shown in Figure 1 and presented in [14]. First, focus group recordings were transcribed (1) by the first author. Then, the transcript and written notes were read multiple times to apply an open coding step (2), yielding 82 codes. For this, we followed the guidelines of Richards et al. [17]. All transcripts and notes were analyzed by the first two authors simultaneously in order to identify patterns. Afterwards, notes were compared and cross-validated where needed. Then, codes were further categorized in an axial coding step (3), yielding 39 subthemes. Multiple revisions of mind maps were made individually and discussed in group to finally arrive at seven main themes (4) after merging results from each focus group session.

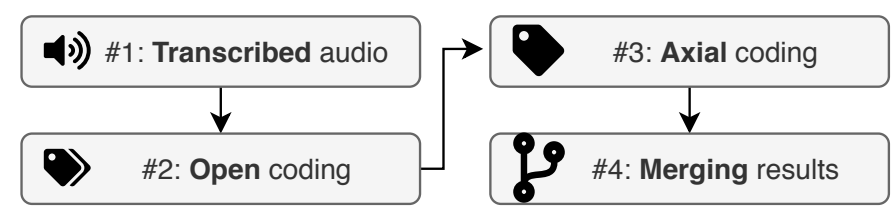

Fig. 1: Focus Group Data Processing setps.

After conducting the second focus group and merging results, the calculated thematic data saturation in accordance with [18] was not yet reached (information threshold: $27 \%$ ). After the fourth session, the threshold dropped below the preset $5 \%$, indicating the end of the data collection phase. This effect is visible in Figure 2 .

We have used an emergent-systematic focus group design [14], in which we organise multiple focus groups to assess if the themes that emerged from one group also emerge from other groups. The full list of the 39 identified subthemes can be consulted in appendix A

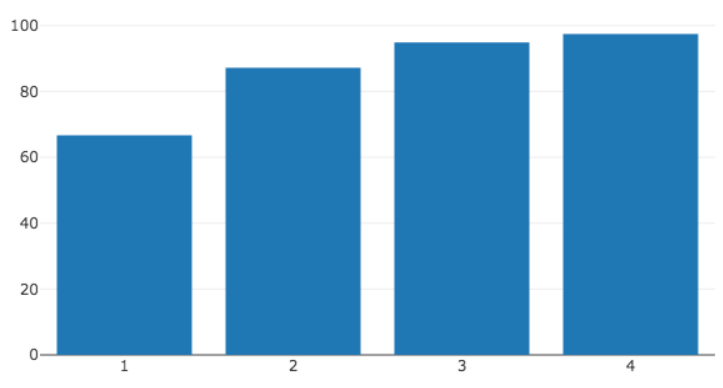

Fig. 2: Data saturation: focus groups (x-axis) projected on the percentage of discovered codes (y-axis). Theme distribution rates are also visible in Table $\mathrm{I}$

\section{RESULTS AND DISCUSSION}

Table I] represents focus group meta-data. A total of 33 participants contributed to 399 minutes of transcripts, yielding a total of 52.329 words. The average number of participants for each session was 8, with an average duration of 100 minutes. Companies involved differed in size, from national to internationally renowned. This, combined with the participant selection process explained in section III led us to believe that respondents have accumulated sufficient experience to be able to contribute to this research.

TABLE I: Focus group meta-data, including theme distribution rate to gauge data saturation.

\begin{tabular}{lrrrr}
\hline Group & Duration & Participants & Words & Theme distr. \\
\hline \hline Total & $399 \mathrm{~m}$ & 33 & 52.329 & $/$ \\
\hline$\# 1$ & $105 \mathrm{~m}$ & 7 & 13.454 & $67 \%$ \\
$\# 2$ & $99 \mathrm{~m}$ & 14 & 12.296 & $87 \%$ \\
$\# 3$ & $100 \mathrm{~m}$ & 7 & 13.492 & $95 \%$ \\
$\# 4$ & $95 \mathrm{~m}$ & 5 & 13.087 & $100 \%$ \\
\hline
\end{tabular}

The seven guidance questions resulted in a lot of similar answers. Therefore, we opted to group themes in subsets of these questions, each of which is briefly discussed. The mind map in Figure 3 was created to provide feedback to respondents on the seven identified categories and 39 subthemes. It was received very positively and deemed complete: no new themes were added.

\section{A. I am (not) creative when I ...}

The warm-up exercise yielded surprisingly uniform results, where developers generally agreed what it means to be creative in their line of work. A few participants tried to define creativity:

\footnotetext{
'When someone can come up with an elegant solution to a previously unsolved non-trivial problem'
} 


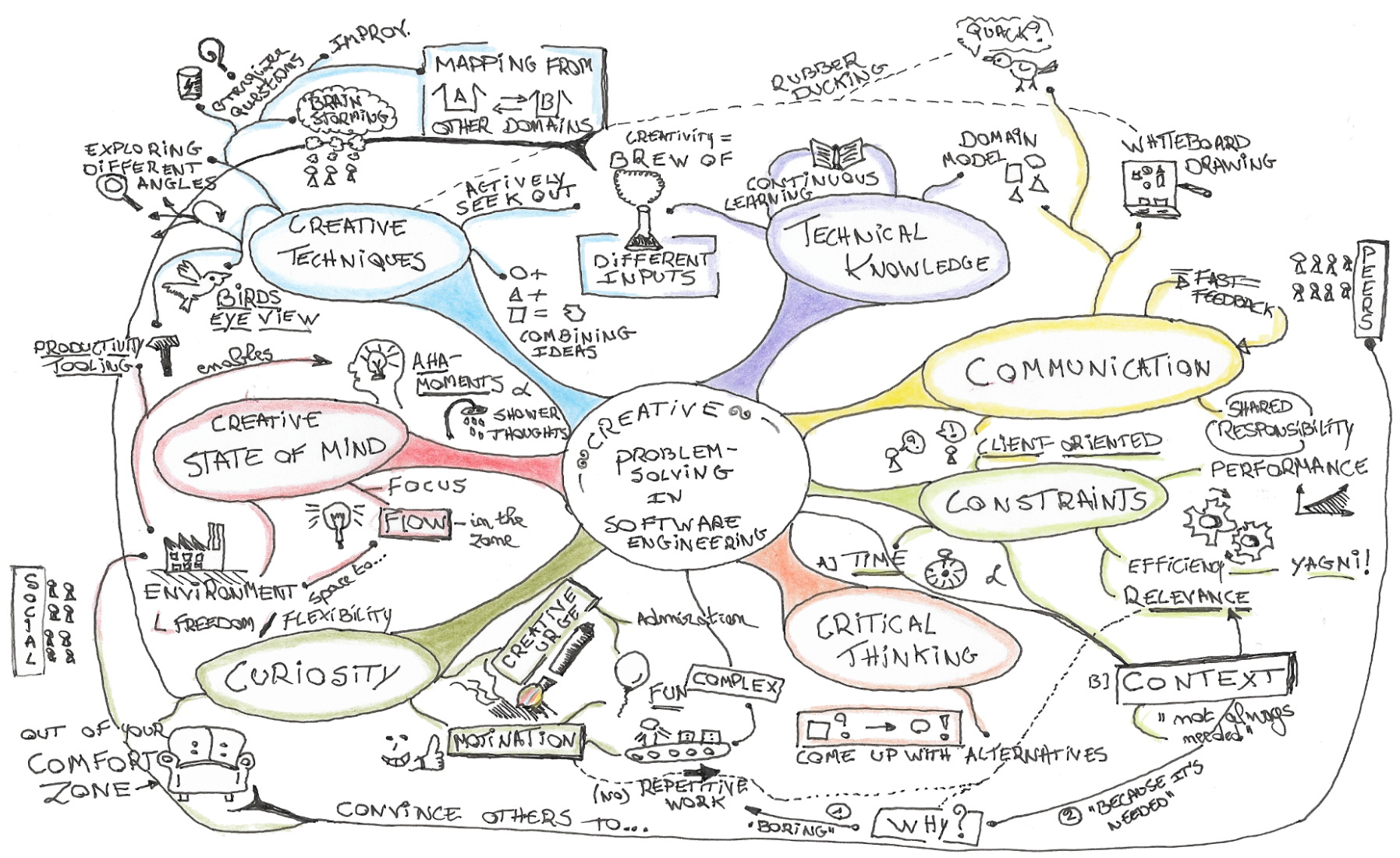

Fig. 3: A mind map that summarizes identified themes on creativity.

However, after a brief discussion, it was criticized: it should be unsolved by the developer, not by anyone, making creativity something inherently personal. Also, it does not need to be elegant: coming up with an inventive quick but ugly hack that works is also deemed as creative. The problem at hand should be complex; otherwise you are simply applying previous knowledge to finish a simple task (cf. Gero's routine design, [7]). Everyone agreed that they are creative when they model or visualize problems, split problems into smaller parts, do complex refactoring work, and brainstorm to approach a problem from another angle.

When are software engineers not so creative? When they blindly take over code without asking anything, when no alternatives are being devised, when tightly defined tasks such as simple TODO lists are being ticked off, when generating boilerplate code, and when repetition kicks in:

'If I just try it until it works, such as fixing dependencies'

Engaging in symptom relief with quick-fixes, rather than addressing the root cause, has likewise been mentioned. Participants also talked about the dangers of tunnel vision, where one is not open to alternatives, consciously or not. Some developers claim that working on legacy code is not creative, while others state that legacy code comes with more constraints that require more creative work compared to maintaining modern projects.

Lastly, interrupts are frequently mentioned as distinctively breaking the creative flow. It has been proven before that 'deep work', being in the flow of work, is of great help in solving problems [19].

\section{B. Requirements to be creative}

In the discussion we identified several requirements or conditions that are necessary for creativity to take place or to improve it. They range from personal traits, such as creative skills and sufficient technical knowledge, to requirements related to the physical, cognitive, and emotional context of work.

1) Personal: technical knowledge and creative skills: There seems to be a thin line between a hack (creative) and fiddling (ignorance). This means that technical knowledge is important, as also denoted by Amabile's Model [8]. Participants indicated that creative work is impossible for them when exploring a new framework or programming language. A firm grasp of the basics is required before being able to do any kind of creative work.

Some participants suggested that creativity says something about the person, not just about the work they do. They wondered whether a person with a big creative drive would 
be creative in any field, given a presence of necessary basic knowledge. Personality correlations with creativity have indeed been confirmed before [20].

Curiosity is cited as an indicator of creativity. A participant suggested that creativity is constantly looking to improve things. Whether or not a person is a continuous improver may be inferred from the frequency of new books on the desk. A 'hungry' mind has been proven before to be a core determinant for achievement [21].

2) Context: physical, cognitive, and emotional: A safe environment, in which developers have the room to express themselves and where failing often is not punished. The more people are penalized for trying out something different, the less new propositions will emerge from them in the future. A lot of participants felt that they are more creative when they deliberately step outside of their comfort zone. This could mean presenting something for a non-technical audience, sharing knowledge, or trying to map problems and solutions outside of their own problem domain to their own. This requires a sufficiently safe environment.

A flexible environment that enables working whenever and wherever they want. This reduces stress and leaves more room for the actual (creative) work - as long as no other mindnumbing activities such as time tracking are required.

An interactive environment. Social interaction, supplemented by drawing material, increases the flow of information, which in turn makes that creative brew even more powerful. Sparring with peers of the same level further eases the exchange of info. Everyone confirmed that higher levels of creativity are reached together than alone - even if interaction is not directly created to creative processes: smalltalk and solutions offered as a joke to entertain each other may just as well lead to more creative work.

An environment that facilitates focus. This includes noisecanceling headphones and places where you can isolate yourself. Some stated they are not bothered by background noise or music while others are easily distracted by it, again hinting at the subjectivity of the circumstances to work creatively. Landscape offices are reported to be both a blessing and a curse: the flow of information is high, as are the amount of unwanted interrupts. Meinel et al. found that designing creativity-enhancing workspaces is by no means a simple task [22].

Leveraging productivity tools allows you to focus on the work itself, letting the tool do everything else such as boilerplating. Tools should match your own thinking process. In one focus group, we accidentally started a Vi versus Emacs war that ended abruptly with one participant saying ' $V i$ won'.

\section{Motivation to be creative}

What motivates developers to be creative? We found several drivers we grouped in three themes: getting rewarded for their work, being cognitively challenged, and a personal need to be creative.

1) Reward: Although not everyone felt this was absolutely necessary, the customer-oriented aspect also plays a certain

role. A solution to a problem should be relevant for end users, and be received positively. As one participant expressed:

'Doing something without feedback is just too
non-committal, like working in the rarefield.'

Customer focus yields more satisfaction, since clients are the ones who provide the constraints on which you can unleash your creativity. Participants also think it is important to be given the responsibility to do major refactoring work. Appreciation for the work from colleagues and from clients is also highly regarded.

2) Challenge: Some developers actively seek out the borders of stepping outside their comfort zone, while others are forced to get creative by having to answer difficult "energizer questions": in both cases they are challenged which leads to creative work.

We noticed a similar importance of being challenged when one participant talked about his creative experience while working on a 'brownfield' legacy conversion project, as opposed to a 'greenfield' project where everything is written from scratch. Everyone agrees that greenfield projects enable creative thinking, since there is room to do things the way you want. However, brownfield projects introduce a lot of constraints, such as a strict budget and a legacy database that needs to be kept online, which in turn triggers more creative work (even though some developers dislike brownfield projects because they are more motivated to work on something brand new). Working with constraints has been mentioned several times during all sessions. Highly constrained conditions generate more ideas and are generally perceived as more inspiring [23].

Similar challenging aspects can be found in Full-stack projects. They are frequently mentioned as the most creative, involving the developer in all of aspects of the design: from back-end database structure to front-end UX design. Seeing the project work as a whole, with all pieces of the puzzle falling into place, seeking out input, and getting out of your comfort zone. These challenging projects usually come equipped with lots of constraints, for example a migration or a hackathon with a limited time frame.

3) Personal need: Because otherwise it gets boring. Many developers love their job precisely because it requires a lot of daily creative work. Assembly line work was frequently mentioned as being horribly dull. Nonetheless, someone suggested that a few days of repetitive work might even stimulate creativity. After mastering something, they start wondering how to automate things just to give in to their creative urge:

'In a sense, creativity can work therapeutically. I wouldn't be here if this work wasn't creative.'

\section{Tools and techniques}

To enable and improve creative work, various tools and techniques are brought forward. They can be categorized under analogies and (external/internal) feedback. 
1) Analogies: Mapping solutions from another domain to your problem field is one of the more intriguing techniques. For instance, if you are developing a virtual communication channel, you could try to investigate how these problems are already solved in the postal system or face-to-face conversations.

2) Feedback: As one participant creatively stated:

'Creativity is the brew of different inputs'

He actively tried to seek out these inputs as much as possible. Here, external feedback can be obtained directly by asking for it, but also indirectly by developing your own knowledge relevant for your work. There are myriad ways to do this, for example:

- Asking for a second opinion from others, not waiting until the code review.

- Regularly reading relevant literature to stay up-to-date.

- Attending knowledge sharing meetings.

- Sparring with peers, getting further together.

Next to external feedback, it is also possible to develop internal, self-reflective feedback, such as:

- Peeling the onion by keeping on asking 'why'.

- Arguing with yourself (rubber ducking).

- Distancing yourself from your thoughts, thereby approaching the problem from other angles.

- Seeking out edge cases like inventing improbable scenarios to undermine your own train of thought.

- Switching gears by zooming out to get the broad picture or zooming in on one specific aspect of the problem.

And of course, brainstorming and modeling the domain on a whiteboard was mentioned countless times.

Taking conscious breaks also came up often. Some developers were familiar with Hunt's "Pragmatic Thinking \& Learning" [24], in which he characterizes the well-known 'shower thoughts' as asynchronous callbacks from $R$-mode thinking. Taking a coffee break or deliberately going to the toilet also seemed to pay off.

\section{E. Creativity as a value for $S E$}

Although creativity is considered an important skill in SE, we found that it is more valued when combined with aspects of work commitment and critical thinking.

1) Combined with commitment: In one group, there was a heated discussion about bug-fixing. Some claim finding the bug is creative work, but not fixing it, while others say it is the other way around. It also seemed to depend on the type of bug, and who caused it. Of course, it takes some time to understand someone else's thought process. Some developers do not like to dig deep if they did not write that piece of code, which is why they dismiss debugging as not very creative, while others who like to get their hands dirty seem to claim the opposite. Similar discussions on starting from scratch or not can be found in 'greenfield' versus 'brownfield' projects: some developers dislike brownfield projects because they are more motivated to work on something brand new. It is clear that the ability to be creative combined with a commitment to 'dig deeper' allows for more successful work outcomes which employers supposedly value more.

Everyone agreed that being creative is a requirement to successfully tackle complex problems. Experts noted that most domain-specific problems are not solved before, as the context and limitations of problems are almost always unique. Some participants felt the need to distinguish themselves by committing to be more creative than others. Others say that creativity happens intuitively:

$$
\begin{aligned}
& \text { 'Creativity simply arises when you are solving } \\
& \text { a problem.' }
\end{aligned}
$$

Many developers are passionate in what they do. They can unleash their creative drive at work by showing craftsmanship. However, participants also mentioned not everyone has this urge, and that is fine too. Developers can also solve certain problems by simply using their experience of previously encountered problems, instead of always trying to be creative. There is a time and place for creativity.

2) Combined with critical thinking: It is important to note that creativity does not always have a positive connotation. One can come up with extremely creative, but completely unusable solutions. Participants emphasize the right combination between creativity and critical thinking, taking into account the context and constraints of the problem:

'Creativity is the means, not the goal.'

\section{F. Measuring creativity}

To jump-start the discussion how to measure creativity, we provided the example of the interview process when applying for a job in the SE industry. How can you measure whether an applicant is creative? According to participants, by gauging the thought process when a problem is presented. We list a selection of the possibilities:

- How are problems approached? Present an impossible scenario and see how far they get in trying to solve it.

- Ask questions about something unfamiliar to the applicant.

- Ask open-ended questions outside of the SE field.

- Do a tunnel vision test: ask to list (unit) test cases besides the usual suspects. Are all edge cases considered?

- Is the applicant a critical thinker, and if so, does he only utter critique or also come up with alternatives?

- The wheel does not always have to be reinvented. Does the applicant know the $D R Y$ and YAGNI principles?

Participants additionally mentioned puzzling games such as Escape Rooms and Black Stories as great ways to test creative thinking. Another option is taking a visual approach by looking at the portfolio of the applicant. However, the general consensus was that it is difficult to measure: all indicators are highly subjective.

The same is true for evaluating whether colleagues are creative. Someone suggested to look at body language. Are 
they happy, and making a lot of jokes? Are they 'in the zone'? A participant rejected that statement, as he claimed one can also be very much in the zone by simply 'sticking stamps'. After some discussion, the conclusion was as follows: do they pause now and then, perhaps thinking? If the pause is too long, they are stuck. If there is no pause, it is likely to be assembly work and not creative work. Measuring productivity (visible, doing) is something else than measuring creativity (invisible, thinking). As one developer stated:

\section{'Most of my creative work happens in the car. When I'm at work, all I have to do is type out the solution in my head.'}

Next to the earlier described curious and 'hungry' mind, encouraging others to think creatively by frequently engaging in the discussion is also seen as an indicator for creativity. According to participants, communication is an important aspect of creative work in SE. Everyone agreed on the following:

'An open-ended question should trigger something in a creative person.'

To conclude, observing certain behavior could be a precondition for creativity, not a guarantee. It is easier to evaluate whether a product is creative than whether the process is creative. When asked if effectiveness is linked to creativity, someone commented that it is not a requirement, however, in $\mathrm{SE}$, effectiveness is usually an important constraint.

\section{LIMITATIONS}

The qualitative nature of this study makes it difficult to provide a unifying definition of creativity applicable to everyone in SE. However, defining creativity was never the goal of this research. By adhering to the methodology and by crossvalidating data, as explained in section III. we believe that the results provide relevant and interesting insights that enlightens the role of creativity.

Also, since creativity is a context sensitive and very personal matter, generalizations prove to be very challenging. This could be mitigated by further reproducing this study in other countries and companies. We are convinced to have collected enough data to contribute to creativity research.

As mentioned by some participants, only creative people are attracted to join the focus groups. We intentionally selected on this intrinsic interest to increase relevance of answers. In this way, we could answer $\mathrm{Q} 1$ and $\mathrm{Q} 2$. However, it is still unclear how much creativity is applied daily in the field of SE. Not every discussed form of creativity $(\mathrm{Q} 1)$ is seen as desirable or as making one developer better than the other (Q2). For example, the creative one who continously looks for new ways without effectively delivering a final product may be a worse developer.

\section{CONCLUSIONS AND FUTURE WORK}

The 33 participants of four focus groups brainstormed about the role of creativity in SE, resulting in seven distinct themes, as displayed in Figure 1: technical knowledge, communication, constraints, critical thinking, curiosity, creative state of mind, and creative techniques. These themes and subthemes also provide insight into how creativity could be measured, the reasons for being creative, and what environment is needed to enhance creative work.

There seem to be different levels of creative drive: some software engineers are more passionate than others, both in their work and beyond. Regarding the domain-specificity of creativity [25], we feel that most techniques could also be applied to architecture for example. Of course, both software design and architecture design share the word design. The creative urge thrives in the unexplored: 'it is the unknown that is the most creative'.

We hope this study is useful for two particular groups. First, for SE practitioners, wo are looking for practical tips on creative problem solving. As discussed in Section IV each of the seven identified creative dimensions can prove to be an effective tool when facing a problem in the SE world. Second, for the computing education community, who aspire to inject more creativity into their curriculum.

Our ultimate goal is to imbue and further encourage the creative urge among undergraduate students in higher education. In future work, we plan to develop a theoretical framework based on this work and cognitive psychology literature to assess the creativity of SE students. We aspire to track and enhance students' progress in creative problem solving using pre- and post-intervention measurements.

\section{DATA AVAILABILITY}

The transcripts of the focus groups are available upon request. All identified codes can be inspected in Appendix A. The scripts used to analyze the codes and generate the data saturation graph of Figure 2 are available on https: //people.cs.kuleuven.be/ wouter.groeneveld/creafocus/.

\section{ACKNOWLEDGMENT}

We wish to thank all participants, who managed to find a few hours time to creatively brainstorm about creativity, for their valuable contributions to this research. Thank you all very much!

\section{APPENDIX}

Table III contains all identified subthemes categorized in seven themes during the coding process, along with the occurrence in each focus group. These themes are also visually summarized in the mind map of Figure 3

\section{REFERENCES}

[1] W. Groeneveld, H. Jacobs, J. Vennekens, and K. Aerts, "Non-cognitive abilities of exceptional software engineers: a delphi study," in Proceedings of the 51st ACM Technical Symposium on Computer Science Education, 2020, pp. 1096-1102.

[2] F. O. Bjørnson and T. Dingsøyr, "Knowledge management in software engineering: A systematic review of studied concepts, findings and research methods used," Information and Software Technology, vol. 50, no. 11, pp. 1055-1068, 2008.

[3] D. K. Simonton, "Creativity: Cognitive, personal, developmental, and social aspects." American psychologist, vol. 55, no. 1, p. 151, 2000. 


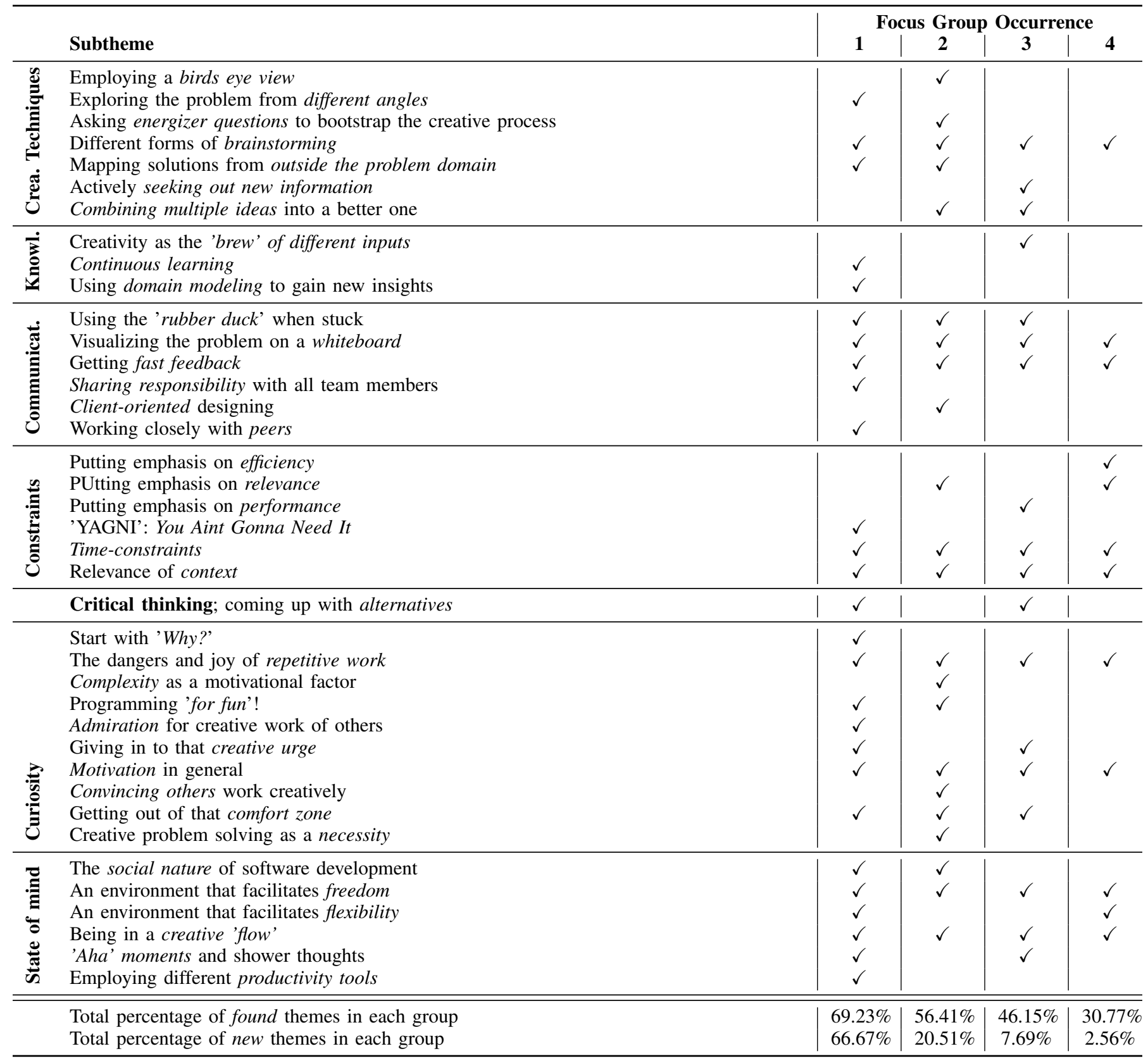

TABLE II: The identified themes and subthemes on creativity, with their occurrences in each conducted focus group. The number of emergent themes drop below the pre-set threshold of $5 \%$ after the fourth session, indicating thematic data saturation.

[4] E. Petkus Jr, "The creative identity: Creative behavior from the symbolic interactionist perspective," The Journal of Creative Behavior, vol. 30, no. 3, pp. 188-196, 1996.

[5] A. Amin, S. Basri, M. F. Hassan, and M. Rehman, "A snapshot of 26 years of research on creativity in software engineering-a systematic literature review," in International Conference on Mobile and Wireless Technology. Springer, 2017, pp. 430-438.

[6] W. Groeneveld, B. A. Becker, and J. Vennekens, "Soft skills: What do computing program syllabi reveal about non-technical expectations of undergraduate students?" in Proceedings of the 2020 ACM Conference on Innovation and Technology in Computer Science Education, 2020, pp. 287-293.

[7] J. S. Gero, "Computational models of innovative and creative design processes," Technological forecasting and social change, vol. 64, no.
2-3, pp. 183-196, 2000.

[8] T. M. Amabile, "A model of creativity and innovation in organizations," Research in organizational behavior, vol. 10, no. 1, pp. 123-167, 1988.

[9] R. L. Mooney, "A conceptual model for integrating four approaches to the identification of creative talent," Scientific creativity: Its recognition and development, pp. 331-340, 1963.

[10] J. Cybulski, L. Nguyen, T. Thanasankit, and S. Lichtenstein, "Understanding problem solving in requirements engineering: Debating creativity with is practitioners," in PACIS 2003: Proceedings of the Seventh Pacific Asia Conference on Information Systems. University of South Australia, 2003, pp. 465-482.

[11] M. Csikszentmihalyi, "Society, culture, and person: A systems view of creativity," in The systems model of creativity. Springer, 2014, pp. 47-61. 
[12] P. L. Li, "What makes a great software engineer," Ph.D. dissertation, 2016.

[13] A. E. Bobkowska, "Exploration of creativity techniques in software engineering in training-application-feedback cycle," in Workshop on Enterprise and Organizational Modeling and Simulation. Springer, 2019, pp. 99-118.

[14] A. J. Onwuegbuzie, W. B. Dickinson, N. L. Leech, and A. G. Zoran, "A qualitative framework for collecting and analyzing data in focus group research," International journal of qualitative methods, vol. 8, no. 3, pp. $1-21,2009$.

[15] J. Kontio, L. Lehtola, and J. Bragge, "Using the focus group method in software engineering: obtaining practitioner and user experiences," in Proceedings. 2004 International Symposium on Empirical Software Engineering, 2004. ISESE'04. IEEE, 2004, pp. 271-280.

[16] E. F. Fern and E. E. Fern, Advanced focus group research. Sage, 2001.

[17] K. A. R. Richards and M. A. Hemphill, "A practical guide to collaborative qualitative data analysis," Journal of Teaching in Physical Education, vol. 37, no. 2, pp. 225-231, 2018.

[18] G. Guest, E. Namey, and M. Chen, "A simple method to assess and report thematic saturation in qualitative research," PLoS One, vol. 15, no. 5, p. e0232076, 2020.

[19] C. Newport, Deep work: Rules for focused success in a distracted world.
Hachette UK, 2016.

[20] M. Reuter, J. Panksepp, N. Schnabel, N. Kellerhoff, P. Kempel, and J. Hennig, "Personality and biological markers of creativity," European Journal of Personality: Published for the European Association of Personality Psychology, vol. 19, no. 2, pp. 83-95, 2005.

[21] S. Von Stumm, B. Hell, and T. Chamorro-Premuzic, "The hungry mind: Intellectual curiosity is the third pillar of academic performance," Perspectives on Psychological Science, vol. 6, no. 6, pp. 574-588, 2011.

[22] M. Meinel, L. Maier, T. Wagner, and K.-I. Voigt, "Designing creativityenhancing workspaces: A critical look at empirical evidence," Journal of technology and innovation management, vol. 1, no. 1, 2017.

[23] M. M. Biskjaer, B. T. Christensen, M. Friis-Olivarius, S. J. Abildgaard, C. Lundqvist, and K. Halskov, "How task constraints affect inspiration search strategies," International Journal of Technology and Design Education, vol. 30, no. 1, pp. 101-125, 2020.

[24] A. Hunt, Pragmatic thinking and learning: Refactor your Wetware. Pragmatic bookshelf, 2008.

[25] P. J. Silvia, J. C. Kaufman, and J. E. Pretz, "Is creativity domainspecific? latent class models of creative accomplishments and creative self-descriptions." Psychology of Aesthetics, Creativity, and the Arts, vol. 3, no. 3, p. 139, 2009. 